\title{
survidm: An R package for Inference and Prediction in an Illness-Death Model
}

by Gustavo Soutinho, Marta Sestelo and Luís Meira-Machado

\begin{abstract}
Multi-state models are a useful way of describing a process in which an individual moves through a number of finite states in continuous time. The illness-death model plays a central role in the theory and practice of these models, describing the dynamics of healthy subjects who may move to an intermediate "diseased" state before entering into a terminal absorbing state. In these models, one important goal is the modeling of transition rates which is usually done by studying the relationship between covariates and disease evolution. However, biomedical researchers are also interested in reporting other interpretable results in a simple and summarized manner. These include estimates of predictive probabilities, such as the transition probabilities, occupation probabilities, cumulative incidence functions, and the sojourn time distributions. The development of survidm package has been motivated by recent contribution that provides answers to all these topics. An illustration of the software usage is included using real data.
\end{abstract}

\section{Introduction}

Multi-state models are very useful for describing complex event history data with multiple endpoints. These models may be considered a generalization of survival analysis where survival is the ultimate outcome of interest but where information is available about intermediate events which individuals may experience during the study period. For instance, in most biomedical applications, besides the 'healthy' initial state and the absorbing 'dead' state, one may observe intermediate (transient) states based on health conditions (e.g., diseased), disease stages (e.g., stages of cancer or HIV infection), clinical symptoms (e.g., bleeding episodes), biological markers (e.g., CD4 T-lymphocyte cell counts; serum immunoglobulin levels), or they can represent a non-fatal complication in the course of the illness (e.g., cancer recurrence, transplantation, etc.). Graphically, these models may be illustrated using diagrams with boxes representing the states and with arrows between the states representing the possible transitions. The complexity of the multi-state model greatly depends on the number of states and also on the possible transitions. The illness-death model is probably the most popular one in the medical literature. The irreversible version of this model (Figure 1) describes the pathway from an initial state to an absorbing state either directly or through an intermediate state. Many event-history data sets from biomedical studies with multiple endpoints can be reduced to this generic structure. There exists extensive literature on multi-state models. Main contributions include books by Andersen et al. (1993) and Hougaard (2000) (Chapter 5 and 6). Recent reviews on this topic may be found in the papers by Putter et al. (2007), Meira-Machado et al. (2009), and Meira-Machado and Sestelo (2019).

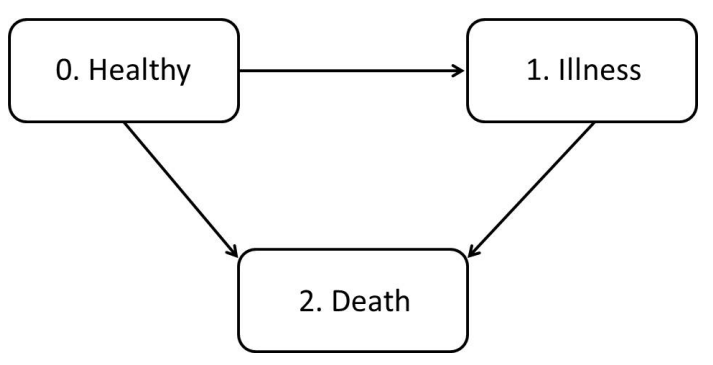

Figure 1: Progressive illness-death model.

One important goal in multi-state modeling is to relate the individual characteristics with the intensity rates through a covariate vector, but biomedical researchers are also interested in reporting interpretable results in a simple and summarized manner. These include estimates of predictive probabilities, such as the transition probabilities, occupation probabilities, cumulative incidence functions, and the sojourn time distributions. The development of survidm $\mathrm{R}$ package has been motivated by several recent contributions that account for these problems; in particular the newly developed methods based on subsampling (see Meira-Machado and Sestelo (2019) for further details). The current version of the package provides seven different approaches to estimate the transition probabilities, two methods for the sojourn distributions and two approaches for the cumulative incidence functions. In addition, these probabilities can also be estimated conditionally on covariate measures. The package also allows the user to perform multi-state regression where the estimation of 
the covariate effects is achieved using Cox regression in which different effects of the covariates are assumed for different transitions.

Several researchers have recently developed software for multi-state survival analysis. A comprehensive list of the available packages in the Comprehensive R Archive Network (CRAN) can be seen in the CRAN task view 'Survival Analysis' (Allignol and Latouche, 2018). In R, several packages provide functions for estimating the transition probabilities (e.g., the package p3state.msm (Meira-Machado and Roca-Pardiñas, 2011), TPmsm (Araújo et al., 2014), etm (Allignol et al., 2011), mstate (de Wreede et al., 2011), and TP.idm (Balboa and de Uña-Álvarez, 2018)), but none implements all the methods addressed by survidm which includes all newly developed methods based on the subsampling approach (see de Uña-Álvarez and Meira-Machado (2015) and references therein). In addition, not all allow the users to obtain estimates of the transition probabilities conditional to covariates. The cmprsk and the timereg $R$ packages can be used to estimate the cumulative incidence functions in a competing risks model. The package survival (via survfit and coxph functions) can also be used for competing risks data. The msSurv can be used to estimate the state occupation probabilities and the sojourn distributions for multi-state models subject to right-censoring (possibly state-dependent) and left-truncation. The package also provides matrices of transition probabilities between any two states. However, none of the available software provides an encompassing package which can be used to estimate all these quantities. Finally, the use of different packages for estimating these quantities separately is rather difficult because each of the current programs requests its own data structure. This paper introduces survidm (available from the Comprehensive R Archive Network at https://cran.r-project.org/web/packages/survidm/), a software application for R which performs inference in a progressive illness-death model. It describes the capabilities of the program for estimating semiparametric regression models and for implementing nonparametric estimators for all quantities mentioned above.

The remainder of this paper is organized as follows. The following section provides a brief introduction to the methodological background. Then, a detailed description of the package is presented, and its usage is illustrated through the analysis of a real data set. Finally, the last section contains the main conclusions of this work.

\section{Methodology background}

The mathematical background underlying the survidm package is briefly introduced in this section. A more detailed introduction can be found in Meira-Machado and Sestelo (2019). The present contribution builds on this article by offering guidelines for using the software to implement the proposed methods.

\section{Notation}

A multi-state model is a model for a time-continuous stochastic process $(Y(t), t \geq 0)$ which at any time occupies one of a few possible states. In this paper, we consider the progressive illness-death model depicted in Figure 1, and we assume that states are numbered as 0 - healthy, 1 - illness, and 2 - death. We also assume that all subjects enter the study in State 0 and that they may either visit State 1 at some time point; or not, going directly to the absorbing state (State 2).

This model is characterized by the joint distribution of $(Z, T)$, where $Z$ denotes the sojourn time in the initial State 0 , and $T$ is the total survival time of the process. As usual with survival data, individuals are generally followed over a certain period of time, providing right-censored observations which are modeled by considering a censoring variable $C$, which we assume to be independent of of $(Z, T)$. Due to censoring, rather than $(Z, T)$, we observe $\widetilde{Z}=\min (Z, C), \widetilde{T}=\min (T, C), \Delta_{1}=I(Z \leq C)$, and $\Delta=I(T \leq C)$ for the respective censoring indicators of $Z$ and $T$. Finally, the available data is $\left(\widetilde{Z}_{i}, \widetilde{T}_{i}, \Delta_{1 i}, \Delta_{i}\right), 1 \leq i \leq n$, i.i.d. copies of $\left(\widetilde{Z}, \widetilde{T}, \Delta_{1}, \Delta\right)$.

\section{Regression models for transitions intensities}

One important goal in multi-state modeling is to study the relationships between the different predictors and the outcome. To relate the individual characteristics to the intensity rates, several models have been used in the literature. A common simplifying strategy is to decouple the whole process into various survival models by fitting separate intensities to all permitted transitions using semiparametric Cox proportional hazard regression models (Cox, 1972), while making appropriate adjustments to the risk set. The most common models are characterized through one of the two model assumptions that can be made about the dependence of the transition intensities and time. The transition intensities may be modeled using separated Cox models assuming the process to be Markovian (also known as 
the clock forward modeling approach), which states that past and future are independent given the present state. They can also be modeled using a semi-Markov model in which the future of the process does not depend on the current time but rather on the duration in the current state. Semi-Markov models are also called 'clock reset' models because each time the patient enters a new state, the time is reset to 0 . The package survidm is restricted to these two semiparametric multi-state models, but other models are possible for the analysis of multi-state survival data. For example, time-homogeneous markov models and model with piecewise constant intensities are implemented in the msm $\mathrm{R}$ package (Jackson, 2011). Aalen additive model (Aalen et al., 2001) and accelerated failure time models (Wei, 1992) are another class of regression models that can be an alternative to the Cox proportional hazards model.

\section{Transition probabilities}

For two states $h, j$ and two time points $s<t$, the so-called transition probabilities $p_{h j}(s, t)=P(Y(t)=$ $j \mid Y(s)=h)$ are introduced. In the progressive illness-death model, there are five different transition probabilities to estimate: $p_{00}(s, t), p_{01}(s, t), p_{02}(s, t), p_{11}(s, t)$, and $p_{12}(s, t)$. Since $p_{00}(s, t)+p_{01}(s, t)+$ $p_{02}(s, t)=1$ and $p_{11}(s, t)+p_{12}(s, t)=1$, in practice, one only needs to estimate three of these quantities. The state occupation probabilities are defined as $p_{j}(t)=P(Y(t)=j)$. If we assume that all subjects are in State 0 at time $t=0$, then $p_{j}(t)=p_{0 j}(0, t)$ and, therefore, the occupation probabilities can be seen as a particular case of the transition probabilities. Estimating these quantities is interesting since they allow for long-term predictions of the process.

The standard nonparametric method to estimate a transition probability matrix is the time-honored Aalen-Johansen (AJ) estimator (Aalen and Johansen, 1978). This estimator benefits from the assumption of Markovianity on the underlying stochastic process extending the time-honored Kaplan-Meier estimator (Kaplan and Meier, 1958) to Markov chains. Explicit formulae of the Aalen-Johansen estimator for the illness-death model are available (Borgan, 1988).

Moreira et al. (2013) propose a modification of the Aalen-Johansen estimator in the illness-death model based on a preliminary smoothing (also known as presmoothing, Dikta (1998); Cao et al. (2005)) of the censoring probability for the total time (respectively, of the sojourn time in State 0), given the available information. The presmoothed Aalen-Johansen (PAJ) estimator proposed by Moreira et al. (2013) is obtained by replacing the censoring indicators (in the transition probabilities $p_{00}(s, t)$ and $\left.p_{11}(s, t)\right)$ by an estimator of a binary (logistic) regression function. The authors verified through simulations that the use of presmoothing can lead to improved estimators with less variability.

The Markov assumption may be violated in practice. For example, for the progressive illnessdeath model, the arrival time to the intermediate state of the process often influences the subsequent transition hazard, leading to non-Markov structures. If the Markov property is violated, then the consistency of the time-honored Aalen-Johansen estimator and of its presmoothed versions can not be ensured in general. Exceptions to this are the estimators for $p_{00}(s, t)$ or for the so-called occupation probabilities, $p_{0 j}(0, t)$ (Datta and Satten, 2001).

Estimators for the transition probabilities in the progressive illness-death model, which do not rely on the Markov assumption, were introduced for the first time by Meira-Machado et al. (2006). The proposed estimators were defined in terms of multivariate Kaplan-Meier integrals with respect to the marginal distributions of $Z$ and $T$. These authors showed the practical superiority of their estimators relative to the Aalen-Johansen in situations in which the Markov condition is strongly violated. However, their proposal has the drawback of requiring that the support of the censoring distribution contains the support of the lifetime distribution. Otherwise, they only report valid estimators for truncated transition probabilities. To avoid this issue, corrected estimators (labeled in this paper as LIDA, the acronym of Lifetime Data Analysis, the journal in which this estimator was published for the first time) were proposed by de Uña-Álvarez and Meira-Machado (2015) for $p_{01}(s, t)$ and $p_{11}(s, t)$.

The paper by de Uña-Álvarez and Meira-Machado (2015) also introduces estimators based on subsampling. The idea behind subsampling, also referred to as landmarking (Van Houwelingen, 2007), is to consider the subset of individuals observed in State $h$ by time $s$. To be specific, given the time point $s$, to estimate $p_{0 j}(s, t)$ for $j=0,1,2$, the landmark analysis is restricted to the individuals observed in State 0 at time $s$. Whereas, to estimate $p_{1 j}(s, t), j=1,2$, the landmark analysis proceeds from the sample restricted to the individuals observed in State 1 at time $s$. The procedure is then based on (differences between) Kaplan-Meier estimators derived from these subsets of the data. These estimators are termed LM in the present paper as well as in the survidm package.

In some cases, subsampling leads to small sample sizes which may result in estimators with high variability. To avoid this problem, a valid approach is to consider a modification of the landmark estimator based on presmoothing (Meira-Machado, 2016). The presmoothed landmark estimators (PLM) are a good alternative in these situations since they give mass to all the event times, including 
the censored observations.

Subsampling was later used by Putter and Spitoni (2018) to derive a landmark Aalen-Johansen estimator (LMAJ) of the transition probabilities. The idea behind the proposed estimator is to use the Aalen-Johansen estimator of the state occupation probabilities derived from those subsets (consisting of subjects occupying a given state at a particular time) for which consistency have already been proved in multi-state models that are not necessarily Markov (Datta and Satten, 2001). In this latter approach, the application of presmoothed estimators (PLMAJ) is possible too.

Also of interest is the estimation of the transition probabilities given a covariate (or a vector of covariates) that is observed for an individual before the individual makes a particular transition of interest. One standard method, particularly well-suited to the setting with multiple covariates, is to consider estimators based on a Cox's regression model (Cox, 1972) fitted marginally to each transition with the corresponding baseline hazard function estimated by the Breslow's method (Breslow, 1972). One alternative and flexible nonparametric approach is to consider local smoothing by means of kernel weights based on local constant (Nadaraya-Watson) regression. Right censoring is handled by applying inverse probability of censoring weighting. This is a fully nonparametric approach which provides flexible effects of the continuous covariates (Meira-Machado et al., 2015; Rodríguez-Álvarez et al., 2016; Meira-Machado and Sestelo, 2019). The two possible approaches are implemented in the survidm package and labeled as breslow and IPCW, respectively.

\section{Cumulative incidence functions}

Another quantity of interest in multi-state modeling is the cause-specific cumulative incidence function, as defined by Kalbfleisch and Prentice (1980). In the illness-death model, two cumulative incidence functions are of particular interest: the cumulative incidence of the illness and the cumulative incidence of dying without the disease. These quantity represents the probability of an individual being or having been diseased at time $t$. One possible estimator for the cause-specific cumulative incidence function in a competing risks setting can be performed using the estimator proposed by Geskus (2011). This estimator based on the subdistribution hazard is obtained by applying the Nelson-Aalen estimator and the product-limit estimator of the disease-free survival. This estimator can also be expressed in terms of the Kaplan-Meier weights of the distribution of $Z$, the sojourn time in State 0 , as introduced in the paper by Meira-Machado and Sestelo (2019). A modification of this estimator based on presmoothing can be introduced to reduce its variability. Both methods are implemented in the survidm package. Estimation methods for the cumulative incidence function conditionally on covariate measures based on local constant (Nadaraya-Watson) regression are also implemented in the package.

\section{Sojourn distributions}

The estimation of the marginal distributions in multi-state modeling is an interesting topic too. In the context of the illness-death model, if the independence assumption between the censoring variable $C$ and the vector of times $(Z, T)$ is assumed, the marginal distribution of the sojourn time in State $0, Z$, can be consistently estimated by the Kaplan-Meier estimator based on the $\left(\widetilde{Z}_{i}, \Delta_{1 i}\right)^{\prime}$ s. Similarly, the distribution of the total time may be consistently estimated by the Kaplan-Meier estimator based on the $\left(\widetilde{T}_{i}, \Delta_{i}\right)^{\prime}$ s. However, the estimation of the marginal distribution of the sojourn time in State 1 is not such a simple issue. Nonparametric estimates for this marginal distribution allowing for state and path-dependent censoring were proposed by Satten and Datta (2002).

\section{survidm in practice}

This section introduces an overview of how the package is structured.

This software enables both numerical and graphical outputs to be displayed for all methods described in the previous section. This software is intended to be used with the $\mathrm{R}$ statistical program (R Core Team, 2019). Our package is composed of 17 functions that allow users to obtain estimates for all proposed methods. Details on the usage of the functions (described in Table 1) can be obtained with the corresponding help pages.

It should be noted that to implement the methods described in the methodology section, one needs the following variables of data: time1, event1, Stime, and event. Covariates can also be included. The variable time 1 represents the sojourn time in State 0 and Stime the total time, whereas event 1 and event are the respective censoring indicators. This means that event 1 will take the value 1 if the subject leaves State 0 and 0 otherwise; event takes value 1 if the subject reaches State 2 and 0 otherwise. 


\begin{tabular}{ll}
\hline Function & Description \\
\hline survIDM & Create a survIDM object. \\
coxidm & Fits proportional hazards regression models for each transition. \\
tprob & Estimation of the transition probabilities. \\
CIF & Estimation of the cumulative incidence functions. \\
sojourn & Nonparametric estimation of the sojourn distribution in the interme- \\
& diate state. \\
autoplot.survIDM & Visualization of survIDM objects with ggplot2 and plotly graphics. \\
plot.survIDM & Plot for an object of class survIDM. \\
print.survIDM & Print for an object of class survIDM. \\
summary.survIDM & Summary for an object of class surviDM. \\
nevents & Counts the number of observed transitions in the multi-state model. \\
markov.test & Performs a test for the Markov assumption. \\
KM & Computes the Kaplan-Meier product-limit of survival. \\
PKM & Computes the presmoothed Kaplan-Meier product-limit of survival. \\
Beran & Computes the conditional survival probability of the response, given \\
& the covariate under random censoring. \\
KMW & Returns a vector with the Kaplan-Meier weights. \\
PKMW & Returns a vector with the presmoothed Kaplan-Meier weights. \\
LLW & Returns a vector with the local linear weights. \\
NWW & Returns a vector with the Nadaraya-Watson weights. \\
\hline
\end{tabular}

Table 1: Summary of functions in the survidm package.

For illustration, we apply the proposed methods to data from a large clinical trial on Duke's stage III patients affected by colon cancer that underwent a curative surgery for colorectal cancer (Moertel et al., 1990). This data set is freely available as part of the $\mathrm{R}$ survival package. The data is also available as part of the $R$ package survidm. Besides the two event times (disease-free survival time and death time) and the corresponding indicator statuses, a vector of covariates including $\mathrm{rx}$ (treatment: Obs(ervation), Lev(amisole), Lev(amisole)+5FU), sex (1 - male), age (years), nodes (number of lymph nodes with detectable cancer), surge (time from surgery to registration: $0=$ short, $1=$ long), adhere (adherence to nearby organs) are also available. The covariate 'recurrence' is the only timedependent covariate, while the other covariates included are fixed. Recurrence can be considered as an intermediate transient state and modeled using the progressive illness-death model with transient states 'alive and disease-free' and 'alive with recurrence', and the absorbing state 'dead'. In the following, we will demonstrate the package capabilities using this data. Below is an excerpt of the data.frame with one row per individual. Individuals were chosen in order to represent all possible combinations of movements among the three states.

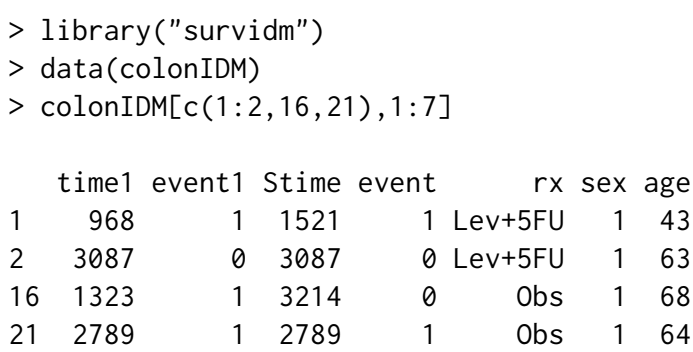

Individual represented in the first line experienced a recurrence of the tumor and have died. In such cases, event $1=1$ and time $1=$ Stime indicate that the individual observed a direct transition from State 0 to State 1 (with event $1=1$ ). Individual represented in line 2 remain alive and without recurrence at the end of follow-up (event $1=0$ and event $=0$ ). Individual represented in line 16 of the original data set, with event $1=1$ and event $=0$, corresponds to an individual with an observed recurrence that remains alive at the end of the follow-up. Note that in this case, the disease-free survival time is equal to the death time (time1 = Stime). Finally, individual represented in line 21 of the original data set has died without observing a recurrence. We note that event $1=1$ and event $=0$ correspond to individuals with an observed recurrence that remain alive at the end of the follow-up.

Of the total of 929 patients, 468 developed a recurrence, and among these 414 died, 38 patients died without developing a recurrence. A summary of the data with the number of the undergoing transitions can be obtained through the nevents function. The colums of the data set must include at least the four columns named time1, event1, Stime, and event according to the requirements of the survIDM function presented in the help file. Parameter state. names enables to change the default 
values of states, 'healthy', 'illness', and 'death'.

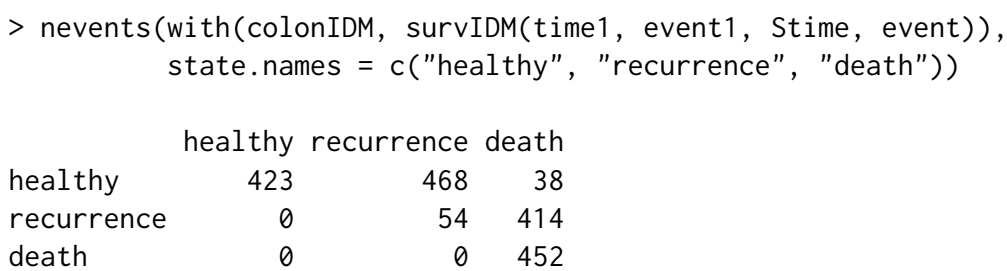

\section{Regression models for transitions intensities}

To relate the individual characteristics to the intensity rates, semiparametric multi-state regression models are used. Specifically, separated Cox models assuming the process to be Markovian (i.e., the transition intensities only depend on the history of the process through the current state) or using a semi-Markov model in which the future of the process does not depend on the current time but rather on the duration in the current state. Therefore, practical interest to determine whether the Markov property holds within a particular data set to determine whether a Markov model or a semi-Markov model is more appropriate.

\section{The Markov assumption}

The Markov assumption may be checked, among others, by including covariates depending on the history. For the progressive illness-death model, the Markov assumption is only relevant for mortality transition after recurrence. We can examine whether the time spent in the initial state "Alive and disease-free" (i.e., the past) is important in the transition from the recurrence state to death (i.e., the future). For doing that, let $Z$ be the time spent in State 0 and $t$ the current time. Fitting a model $\alpha_{12}(t ; Z)=\alpha_{12,0}(t) \exp \{\beta Z\}$, we now need to test the null hypothesis, $H_{0}: \beta=0$, against the general alternative, $H_{1}: \beta \neq 0$. This would assess the assumption that the transition rate from the disease state into death is unaffected by the time spent in the previous state.

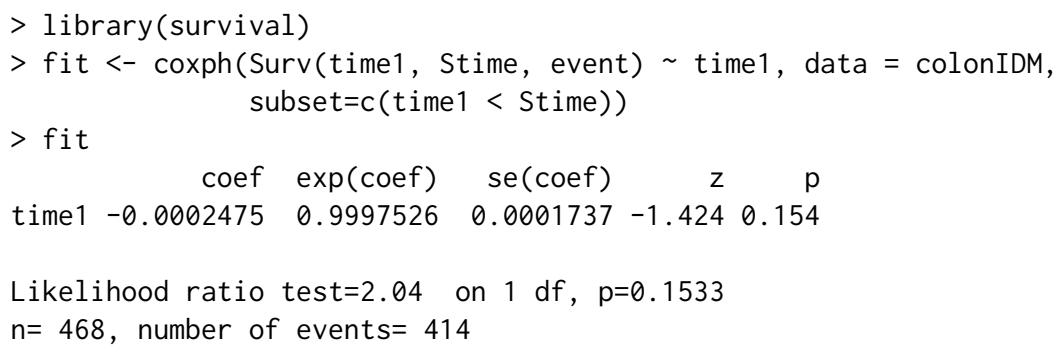

Following this procedure, we verified that the effect of time spent in State 0 reported a $p$-value of 0.154 (regression coefficient: - 0.0002475), revealing no evidence against the Markov model for the colon data. Results from this test can also be obtained through the function markov. test, which has an output fairly similar to those obtained from coxph function.

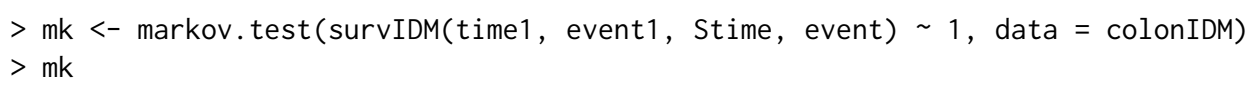

Since there is no evidence on the lack of Markovianity, a multi-state Markov regression model based on the Cox model can be fitted through the following input command:

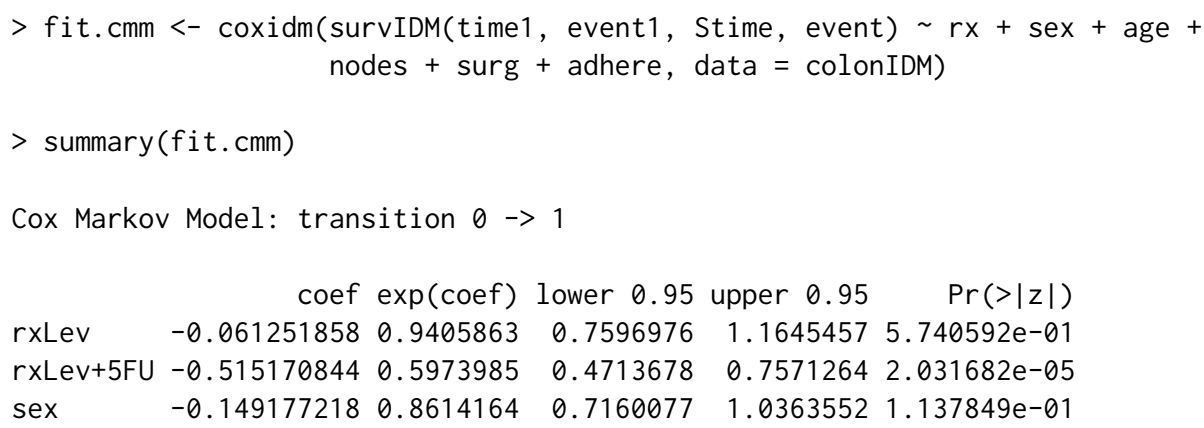




$\begin{array}{lrllll}\text { age } & -0.004669254 & 0.9953416 & 0.9876802 & 1.0030625 & 2.362711 \mathrm{e}-01 \\ \text { nodes } & 0.083943790 & 1.0875678 & 1.0686993 & 1.1067694 & 5.418662 \mathrm{e}-21 \\ \text { surg } & 0.251798521 & 1.2863368 & 1.0509673 & 1.5744186 & 1.460249 \mathrm{e}-02 \\ \text { adhere } & 0.296839791 & 1.3455997 & 1.0551768 & 1.7159575 & 1.671466 \mathrm{e}-02\end{array}$

Cox Markov Model: transition $0 \rightarrow 2$

coef $\exp$ (coef) lower 0.95 upper $0.95 \quad \operatorname{Pr}(>|z|)$

$\begin{array}{lrrrrr}\text { rxLev } & -0.29152482 & 0.7471235 & 0.3271685 & 1.706135 & 4.889711 \mathrm{e}-01 \\ \text { rxLev+5FU } & -0.11211853 & 0.8939383 & 0.4220165 & 1.893589 & 7.697006 \mathrm{e}-01 \\ \text { sex } & 0.39293182 & 1.4813174 & 0.7641923 & 2.871399 & 2.445966 \mathrm{e}-01 \\ \text { age } & 0.08422764 & 1.0878765 & 1.0476871 & 1.129608 & 1.157046 \mathrm{e}-05 \\ \text { nodes } & 0.07538428 & 1.0782984 & 0.9895116 & 1.175052 & 8.552937 \mathrm{e}-02 \\ \text { surg } & 0.41564547 & 1.5153485 & 0.7703441 & 2.980851 & 2.285509 \mathrm{e}-01 \\ \text { adhere } & 0.05435239 & 1.0558566 & 0.4377875 & 2.546517 & 9.036879 \mathrm{e}-01\end{array}$

Cox Markov Model: transition $1->2$

\begin{tabular}{lrrrrr} 
& \multicolumn{1}{c}{ coef } & $\exp ($ coef) & lower 0.95 & upper 0.95 & $\operatorname{Pr}(>|z|)$ \\
rxLev & 0.068953592 & 1.071386 & 0.8533466 & 1.345138 & $5.525534 \mathrm{e}-01$ \\
rxLev+5FU & 0.327043851 & 1.386862 & 1.0741245 & 1.790656 & $1.212756 \mathrm{e}-02$ \\
sex & 0.214094887 & 1.238740 & 1.0138220 & 1.513557 & $3.623833 \mathrm{e}-02$ \\
age & 0.009342474 & 1.009386 & 1.0014760 & 1.017359 & $1.994502 \mathrm{e}-02$ \\
nodes & 0.046061552 & 1.047139 & 1.0249376 & 1.069821 & $2.522475 \mathrm{e}-05$ \\
surg & -0.012258877 & 0.987816 & 0.7944594 & 1.228232 & $9.121722 \mathrm{e}-01$ \\
adhere & 0.137708158 & 1.147641 & 0.8851963 & 1.487895 & $2.985854 \mathrm{e}-01$
\end{tabular}

The transition intensities characterize the hazard for movement from one state to another, revealing how the different covariates affect the various permitted transitions. The results obtained indicate that none of the covariates were found to have a strong effect on all three transitions. Save for covariates age and sex, all the remaining predictors were considered important for recurrence transition. Interestingly, age displayed a strong linear effect on mortality transition without recurrence, whereas all the other covariates failed to show relevant association on this transition. Finally, save for covariates surg and adhere, all the remaining predictors were considered important for the mortality transition after recurrence. The coxidm function also returns the analysis of the deviance for each Cox model. In this case, only an overall $p$-value is presented for categorical variables. To obtain the outputs, we have to indicate type='anova' in summary function.

$>\operatorname{summary}($ fit.. $\mathrm{cmm}$, type $=$ 'anova')

Cox Markov Model: transition $0 \rightarrow 1$

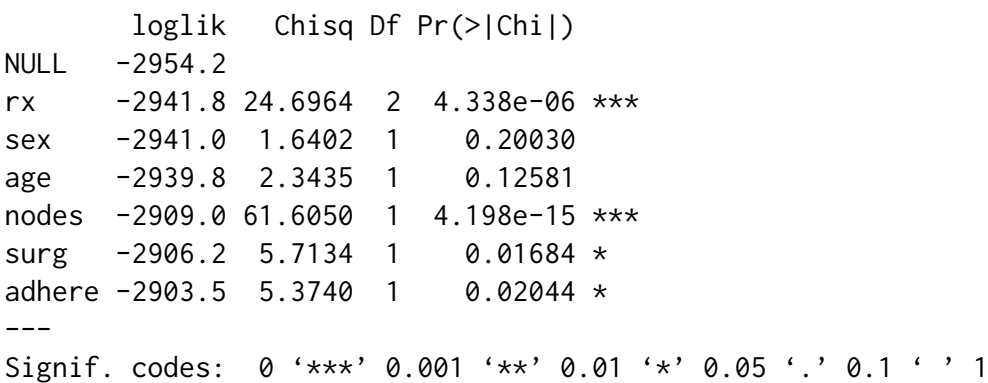

Cox Markov Model: transition $0 \rightarrow 2$

$\begin{array}{lrrrc} & \text { loglik } & \text { Chisq } & \text { Df } & \operatorname{Pr}(>\mid \text { Chi } \mid) \\ \text { NULL } & -231.79 & & & \\ \text { rx } & -231.54 & 0.4938 & 2 & 0.7812 \\ \text { sex } & -231.04 & 1.0065 & 1 & 0.3158 \\ \text { age } & -219.26 & 23.5445 & 1 & 1.221 \mathrm{e}-06\end{array}$ ***




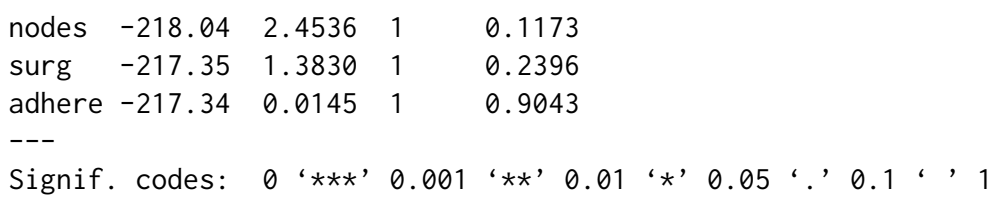

Cox Markov Model: transition $1 \rightarrow 2$

\begin{tabular}{|c|c|c|c|c|}
\hline & loglik & Chisq & Df $\operatorname{Pr}(>\mid$ Chi $\mid)$ & \\
\hline NULL & -1897.5 & & & \\
\hline$r x$ & -1895.0 & 4.8864 & 0.0868804 & . \\
\hline $\operatorname{sex}$ & -1892.8 & 4.3995 & 0.0359501 & * \\
\hline age & -1890.8 & 4.0650 & 0.0437799 & * \\
\hline nodes & -1883.4 & 14.7205 & 0.0001247 & $\star \star \star$ \\
\hline surg & -1883.4 & 0.0090 & 0.9242629 & \\
\hline adhere & -1882.9 & 1.0505 & 10.3054007 & \\
\hline
\end{tabular}

The effect of the continuous covariates on the log hazards is often assumed to have a linear functional form in all intensities. To introduce flexibility into the Cox Markov model, several smoothing methods may be applied, but P-splines (Eilers and Marx, 1996) are being most frequently considered in this context. Results showed a strong nonlinear effect for nodes (checked through a formal test) when using a Cox model on the recurrence transition. Figure 2 returns a centered set of predictions on a log hazard scale. The average predicted value is zero with a mean value of nodes as the reference (see the vignette 'Splines, plots, and interactions' in (Therneau, 2021)). The main curve depicts the smooth curve for nodes on a log hazard scale, indicating that the risk of recurrence increases rapidly until about 6 nodes. The apparent decrease after 23 nodes is not significant due to the wide confidence intervals.

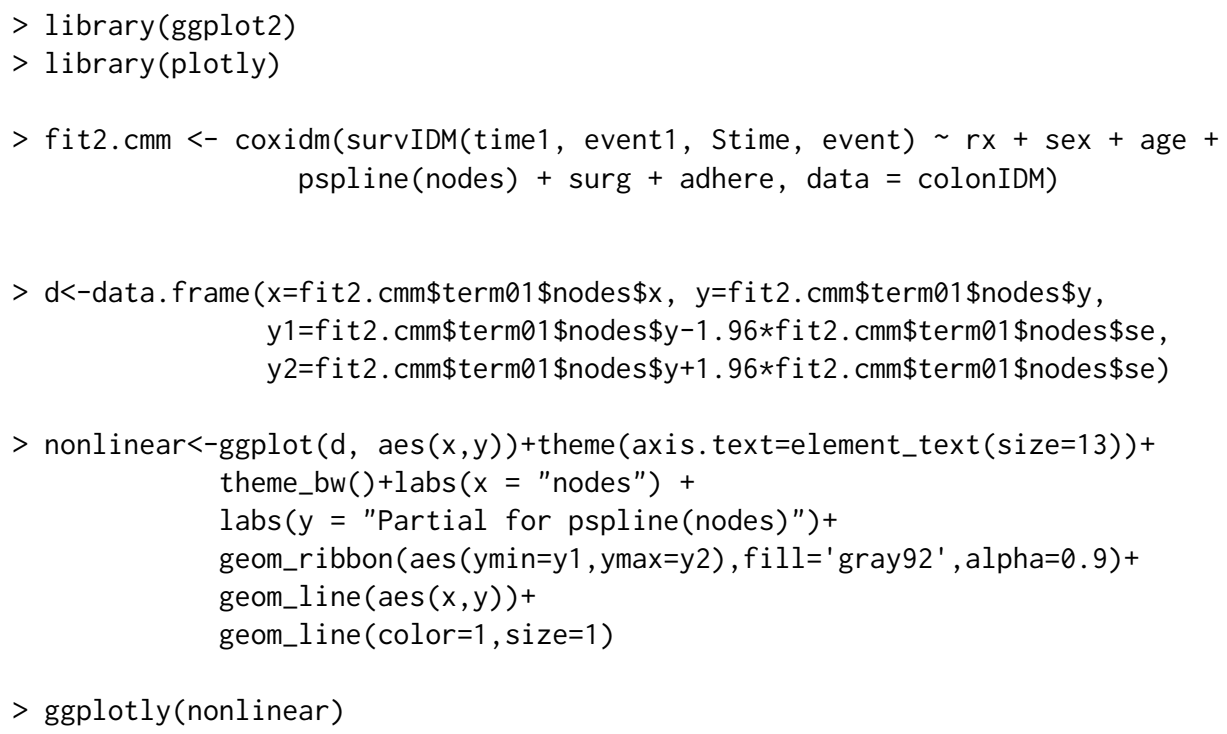

The proportional hazards assumption can be tested formally using the summary function. The output can be obtained putting type=' $\mathrm{ph}$ ' in summary function.

$>\operatorname{summary}\left(\right.$ fit2. $\mathrm{cmm}$, type $\left.={ }^{\prime} \mathrm{ph}^{\prime}\right)$

Cox Markov Model: transition $0 \rightarrow 1$

Test the Proportional Hazards Assumption

$\begin{array}{lrrr} & \text { chisq } & d f & p \\ r x & 4.12 e-01 & 2.00 & 0.81 \\ \text { sex } & 2.10 e+00 & 1.00 & 0.15 \\ \text { age } & 9.37 e-04 & 1.00 & 0.98\end{array}$




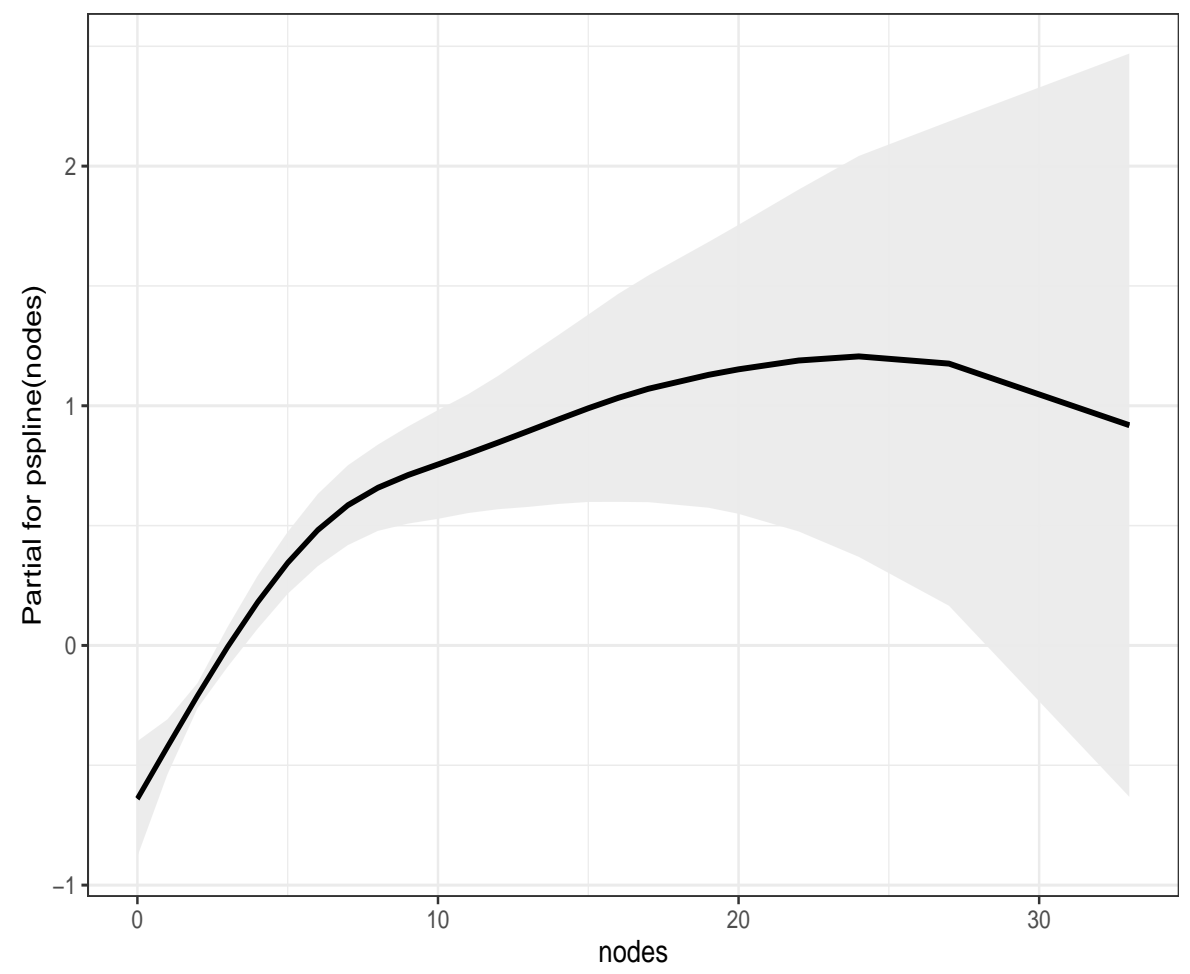

Figure 2: Predicted values of the smooth log hazard based on penalized splines (black line) with pointwise $95 \%$ confidence intervals obtained from the partial residuals for nodes (recurrence intensity), using the colon cancer data.

$\begin{array}{llll}\text { pspline(nodes) } & 7.60 \mathrm{e}+00 & 3.95 & 0.10 \\ \text { surg } & 1.97 \mathrm{e}+00 & 1.00 & 0.16 \\ \text { adhere } & 6.13 \mathrm{e}-01 & 1.00 & 0.43 \\ \text { GLOBAL } & 1.30 \mathrm{e}+01 & 9.94 & 0.22\end{array}$

Cox Markov Model: transition $0 \rightarrow 2$

Test the Proportional Hazards Assumption

$\begin{array}{lccc} & \text { chisq } & \text { df } & p \\ \text { rx } & 1.6292 & 2.00 & 0.44 \\ \text { sex } & 0.0668 & 1.00 & 0.80 \\ \text { age } & 0.8396 & 1.00 & 0.36 \\ \text { pspline(nodes) } & 0.7859 & 4.00 & 0.94 \\ \text { surg } & 0.4955 & 1.00 & 0.48 \\ \text { adhere } & 2.3606 & 1.00 & 0.12 \\ \text { GLOBAL } & 6.1424 & 9.99 & 0.80\end{array}$

Cox Markov Model: transition $1 \rightarrow 2$

Test the Proportional Hazards Assumption

$\begin{array}{lrrr} & \text { chisq } & d f & p \\ \text { rx } & 5.03913 & 1.99 & 0.08 \\ \text { sex } & 0.02204 & 1.00 & 0.88 \\ \text { age } & 0.73628 & 1.00 & 0.39 \\ \text { pspline(nodes) } & 4.25500 & 4.09 & 0.39 \\ \text { surg } & 2.02427 & 1.00 & 0.15 \\ \text { adhere } & 0.00177 & 1.00 & 0.97 \\ \text { GLOBAL } & 13.19170 & 10.08 & 0.22\end{array}$

A semi-Markov model could be obtained by including the argument semiMarkov = TRUE in the 
coxidm function.

\section{Occupation probabilities and transition probabilities}

The occupation probabilities and the transition probabilities are key quantities of interest in multi-state models. They offer interpretable results in a simple and summarized manner.

Estimates and plots of the transition probabilities for all methods introduced in Section 2.2 can be obtained using the tprob function. The default method is the Aalen-Johansen estimator (AJ) which assumes the process to be Markovian. The presmoothed version of the Aalen-Johansen estimator (PAJ) also assumes the process to be Markovian while the remaining methods (LIDA, LM, PLM, LMAJ, and PLMAJ) are free of the Markov condition.

When one is confident of the Markov assumption, the Aalen-Johansen is preferred over the nonMarkovian estimators since it reports a smaller variance in estimation. Estimates and plot for the Aalen-Johansen method can be obtained through the following input commands:

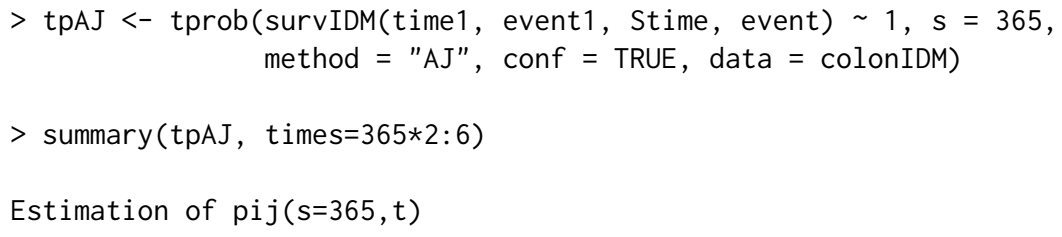

Besides being consistent regardless the Markov condition, the landmark non-Markov estimators (LM, PLM, LMAJ, and PLMAJ) can be preferable in many situations due to their greater accuracy (smaller bias). When comparing the original nonparametric landmark estimator (LM) and the Aalen-Johansen estimator, some discrepancies are observed for $t=730$ and $t=1095$ ( 2 and 3 years, respectively). In addition to the aforementioned discrepancy between the two estimates, the plots for the two methods (Figure 3) also show that the confidence bands are narrower in the case of the Aalen-Johansen, revealing less variability for this method.

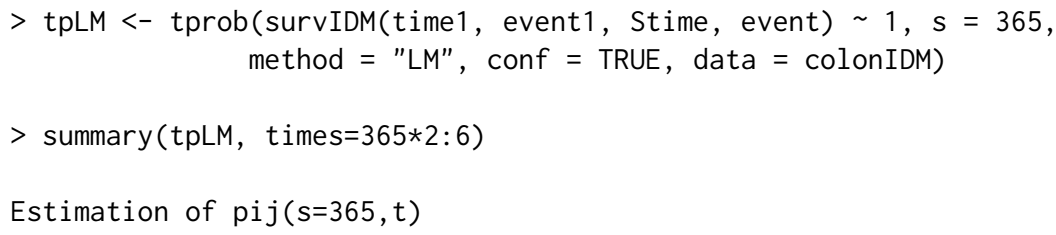



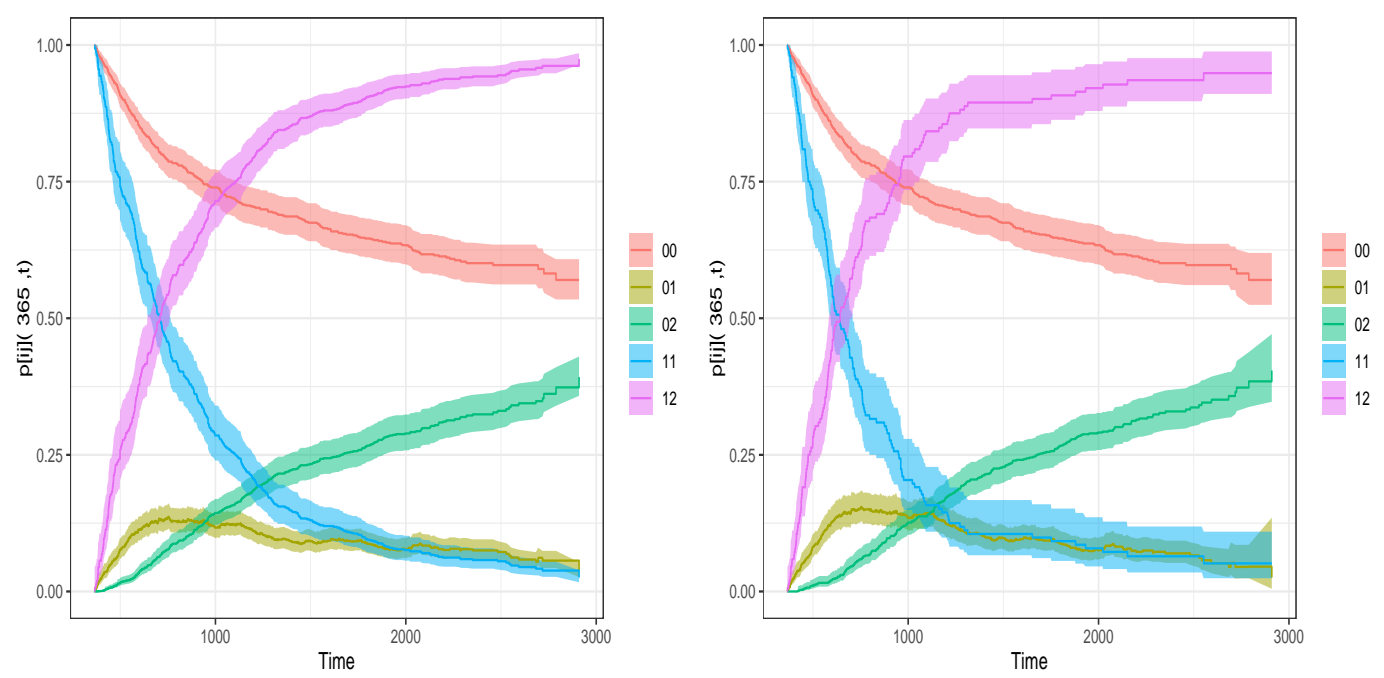

Figure 3: Transition probability estimates using the AJ (left hand side) and LM (right hand side) method, using the colon cancer data.

$\begin{array}{rrrrrr}t & 00 & 01 & 02 & 11 & 12 \\ 730 & 0.7966309 & 0.14750103 & 0.0558681 & 0.38815789 & 0.6118421 \\ 1095 & 0.7192603 & 0.14320925 & 0.1375305 & 0.15789474 & 0.8421053 \\ 1460 & 0.6805333 & 0.09446864 & 0.2249981 & 0.10526316 & 0.8947368 \\ 1825 & 0.6444157 & 0.08583643 & 0.2697479 & 0.09210526 & 0.9078947 \\ 2190 & 0.6131533 & 0.07465238 & 0.3121944 & 0.06432749 & 0.9356725\end{array}$

$2.5 \%$

$\begin{array}{lllllr}t & 00 & 01 & 02 & 11 & 12\end{array}$

$\begin{array}{lllllll}730 & 0.7673274 & 0.12294665 & 0.0411836 & 0.31792669 & 0.5390734\end{array}$

$\begin{array}{llllllll}1095 & 0.6866872 & 0.12033558 & 0.1142137 & 0.10937624 & 0.7860868\end{array}$

$\begin{array}{llllllll}1460 & 0.6468058 & 0.07447488 & 0.1960521 & 0.06621973 & 0.8472552\end{array}$

$\begin{array}{llllllll}1825 & 0.6098421 & 0.06804756 & 0.2387239 & 0.05591405 & 0.8630680\end{array}$

$\begin{array}{lllllll}2190 & 0.5777125 & 0.05742370 & 0.2791810 & 0.03480413 & 0.8969820\end{array}$

$97.5 \%$

$\begin{array}{rrrrrr}\mathrm{t} & 00 & 01 & 02 & 11 & 12 \\ 730 & 0.8270534 & 0.17695930 & 0.07578852 & 0.4739034 & 0.6944337 \\ 1095 & 0.7533784 & 0.17043081 & 0.16560740 & 0.2279357 & 0.9021157 \\ 1460 & 0.7160195 & 0.11982998 & 0.25821767 & 0.1673268 & 0.9448794 \\ 1825 & 0.6809493 & 0.10827565 & 0.30480372 & 0.1517218 & 0.9550498 \\ 2190 & 0.6507682 & 0.09705015 & 0.34911161 & 0.1188947 & 0.9760319 \\ \text { > autoplot(tpLM) } & & & & \end{array}$

Since the landmark estimators of the transition probabilities are free of the Markov assumption, they can also be used to introduce such tests (at least in the scope of the illness-death model) by measuring their discrepancy to Markovian estimators. The function markov. test performs a local graphical test for the Markov condition. This graphical test is based on a PP-plot which compares the estimations reported by the Aalen-Johansen transition probabilities to their non-Markov counterparts. The corresponding plot for a local test of Markovianity $(s=365)$ can be obtained through the following input command:

$>\mathrm{mk}<-$ markov.test(survIDM(time1, event1, Stime, event) $\sim 1, \mathrm{~s}=365$, data $=$ colonIDM) $>$ autoplot (mk)

The plot shown in Figure 4 compares the Aalen-Johansen estimator and the landmark nonMarkovian estimator for $p_{01}(s, t), p_{02}(s, t)$, and $p_{12}(s, t)$, for $s=365$. Existing deviations of the plots with respect to the straight line $y=x$ reveals some evidence on the lack of Markovianity of the underlying process beyond one year after surgery. For further illustration, this figure jointly displays 

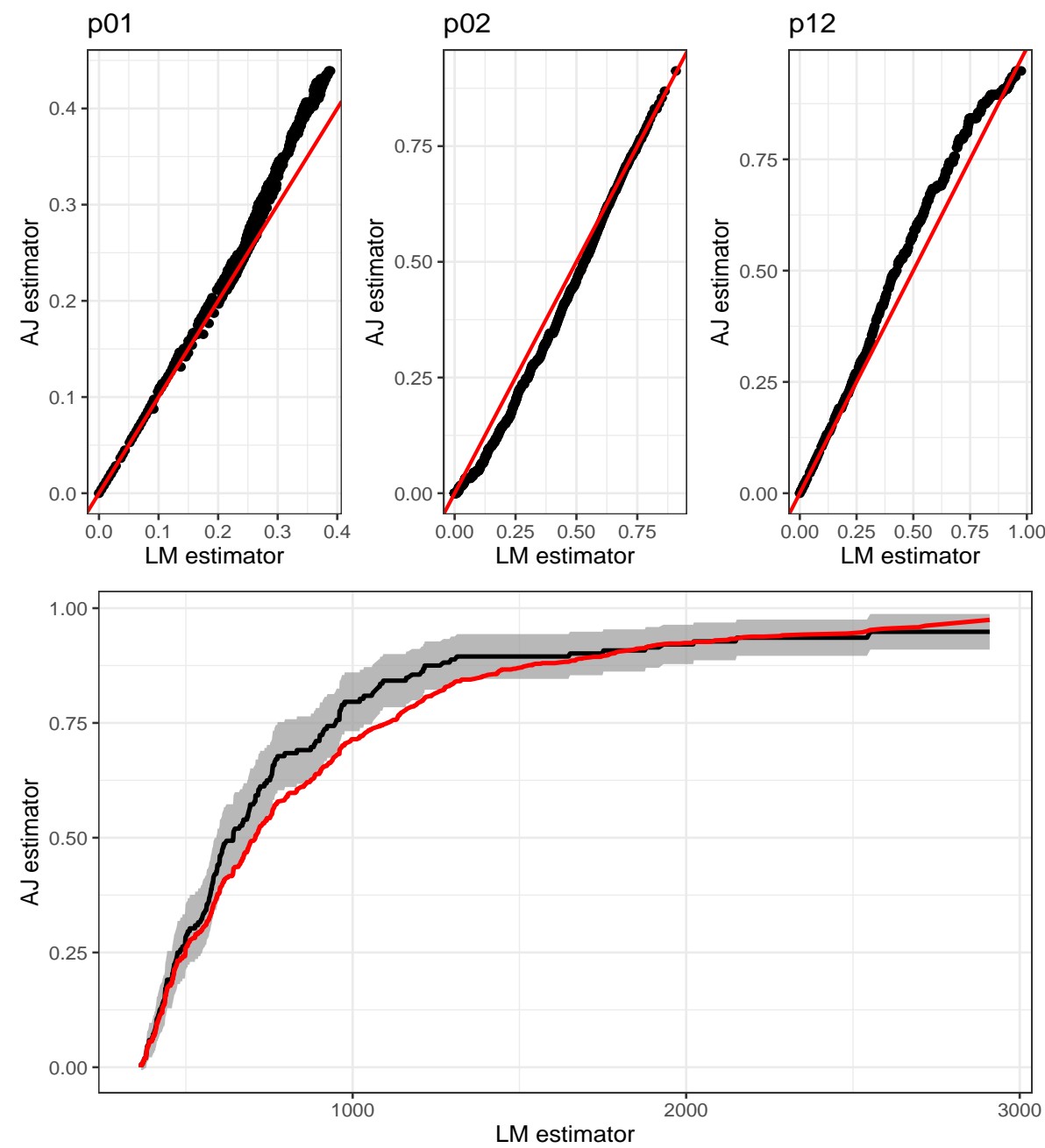

Figure 4: Graphical test for the Markov condition, $s=365$. The second row shows the landmark (Markov-free) estimator with $95 \%$ pointwise confidence limits (black line) and Aalen-Johansen estimator (red line) for the transition probability $p_{12}(365, t)$, using the colon cancer data.

the landmark non-Markovian estimator and the Aalen-Johansen estimator for $p_{12}(s=365, t)$. In this plot, the differences between both estimators are clearly seen. Thus, in principle, the application of the Aalen-Johansen method is not recommended here due to possible biases.

The variability of the nonparametric landmark estimator (LM) may be successfully reduced using presmoothing ideas (Dikta, 1998; Cao et al., 2005). The presmoothed landmark estimator is implemented in the same function through the method PLM. The same ideas can be used to reduce the variability of the Markovian Aalen-Johansen estimator and the (non-Markov) Landmark AalenJohansen estimator through methods PAJ and PLMAJ, respectively.

The package survidm also allows for the computation of the above quantities conditional on covariates that are observed for an individual before the individual makes a particular transition of interest. For continuous covariates, one possible and flexible nonparametric approach is to consider local smoothing by means of kernel weights based on local constant (Nadaraya-Watson: NW) regression. This estimator is implemented in our package through function tprob using the method $=$ IPCW. Below are the input commands to obtain the estimates of the transition probabilities at time $s=365$ for an individual of 48 years old. For the bandwidth in the estimator, we use dpik function, which is available from the R KernSmooth package. This is the data-based bandwidth selector of Wand and Jones (1997). 


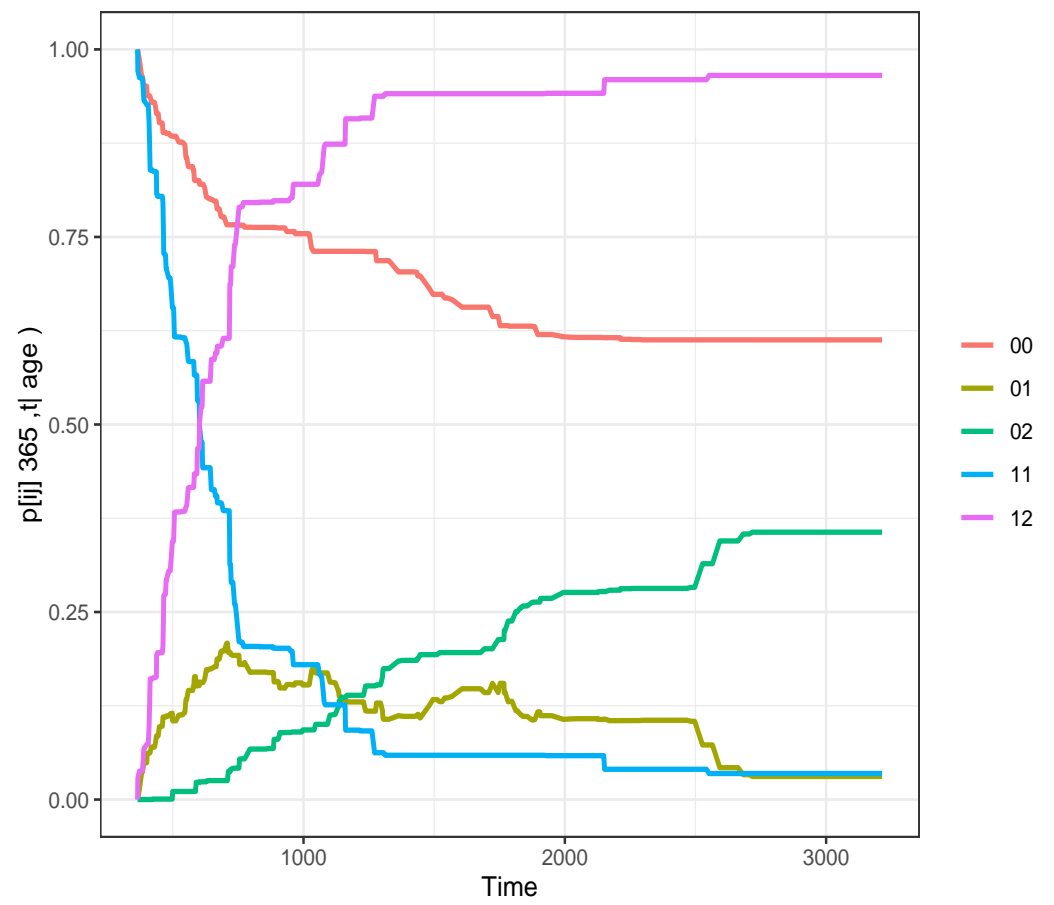

Figure 5: Conditional transition probabilities given that the subject is alive and disease-free at $s=365$ days for a 48-years-old patient, using the colon cancer data.

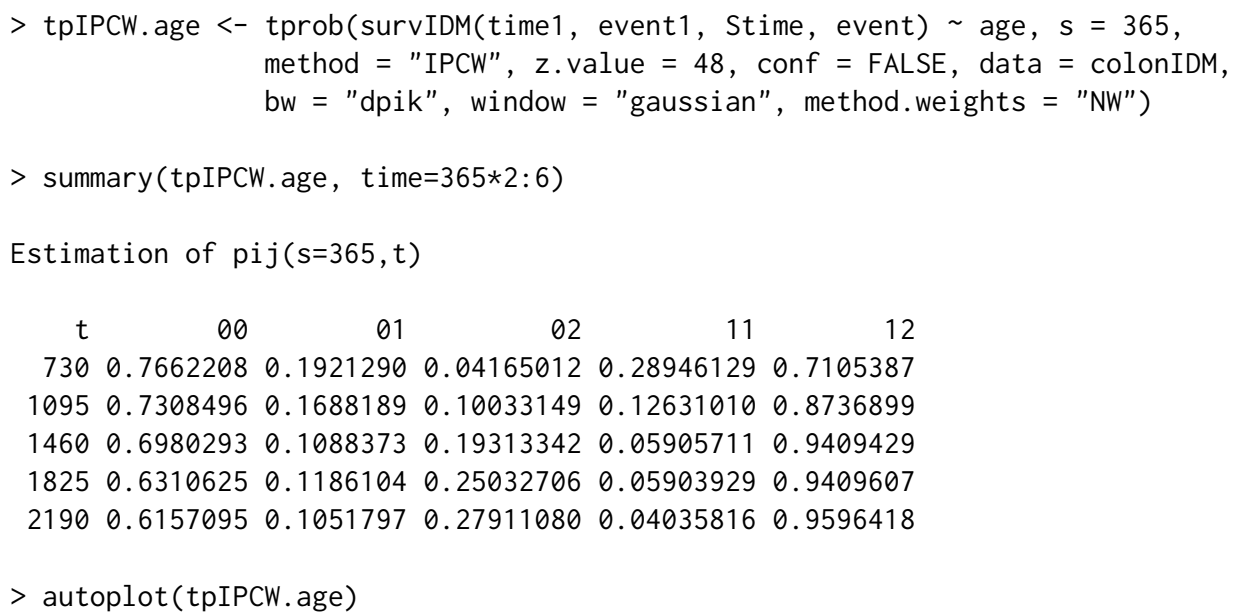

The curves depicted in Figure 5, which are purely nonparametric, enable flexible modeling of the data providing flexible and robust effects of the covariate that can be used at least as a preliminary attempt, providing insights on the data being analyzed. Such methods can be used to capture nonstandard data features that may not be detected through parametric or semiparametric proposals. A general problem in multivariate nonparametric regression estimation is the so-called curse of dimensionality. In higher dimensions, the observations are sparsely distributed even for large sample sizes. Consequently, estimators based on local averaging (like those based on kernel smoothing) perform unsatisfactorily in this situation.

An alternative method is to consider estimators based on Cox's regression model (Cox, 1972) fitted marginally to each transition with the corresponding baseline hazard function estimated by Breslow's method (Breslow, 1972). The following input commands illustrate the use of the tprob function in this context:

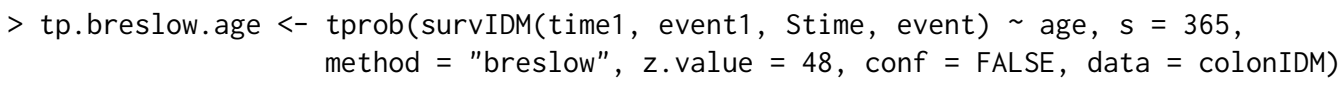


Estimation of $\operatorname{pij}(s=365, t)$

$\begin{array}{rrrrrr}\mathrm{t} & 00 & 01 & 02 & 11 & 12 \\ 730 & 0.7970855 & 0.15020199 & 0.05271253 & 0.37528949 & 0.6247105 \\ 1095 & 0.7198657 & 0.14999685 & 0.13013746 & 0.14814634 & 0.8518537 \\ 1460 & 0.6826444 & 0.10384005 & 0.21351550 & 0.09843946 & 0.9015605 \\ 1825 & 0.6451532 & 0.09850122 & 0.25634562 & 0.08617378 & 0.9138262 \\ 2190 & 0.6139465 & 0.08891388 & 0.29713961 & 0.06066618 & 0.9393338\end{array}$

Note that if the argument $z$. value is missing, then the tprob function computes the predicted conditional transition probabilities at the average values of the covariate. The Breslow method (based on the Cox regression model) is particularly well-suited to the setting with multiple covariates:

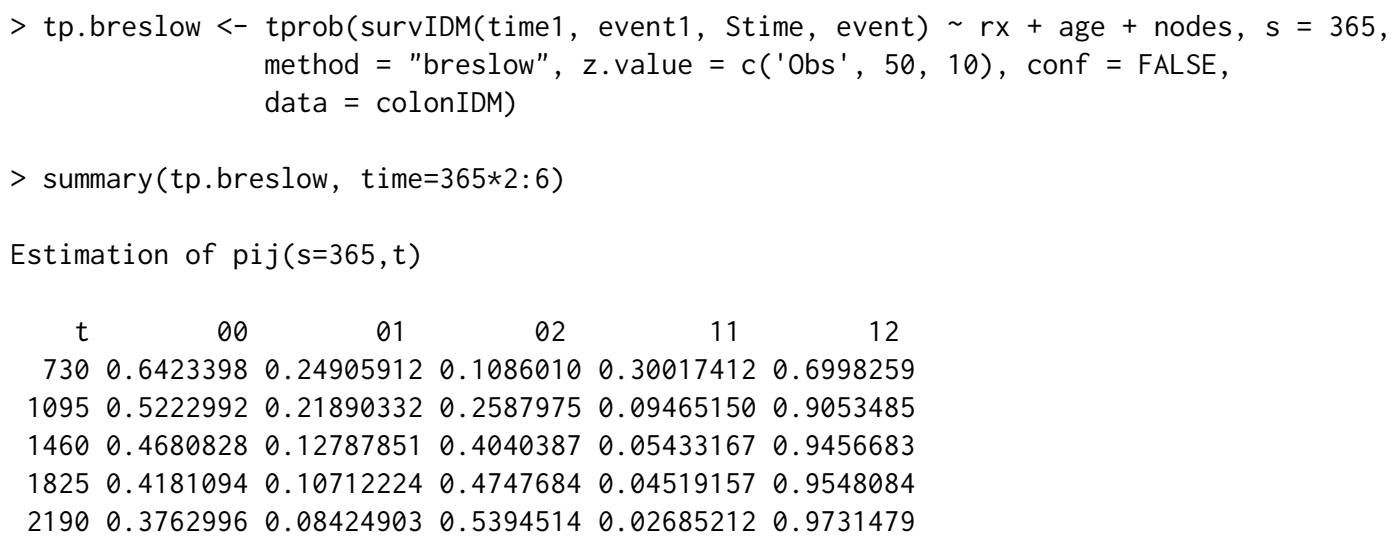

\section{Cumulative Incidence Function}

Another quantity of interest in multi-state modeling is the cause-specific cumulative incidence of the illness (recurrence). Function CIF can be used to obtain the nonparametric estimator of Geskus (2011) (default method), which is equivalent to the classical Aalen-Johansen estimator. The corresponding presmoothed version (Meira-Machado and Sestelo, 2018) is also implemented through the argument presmooth = TRUE:

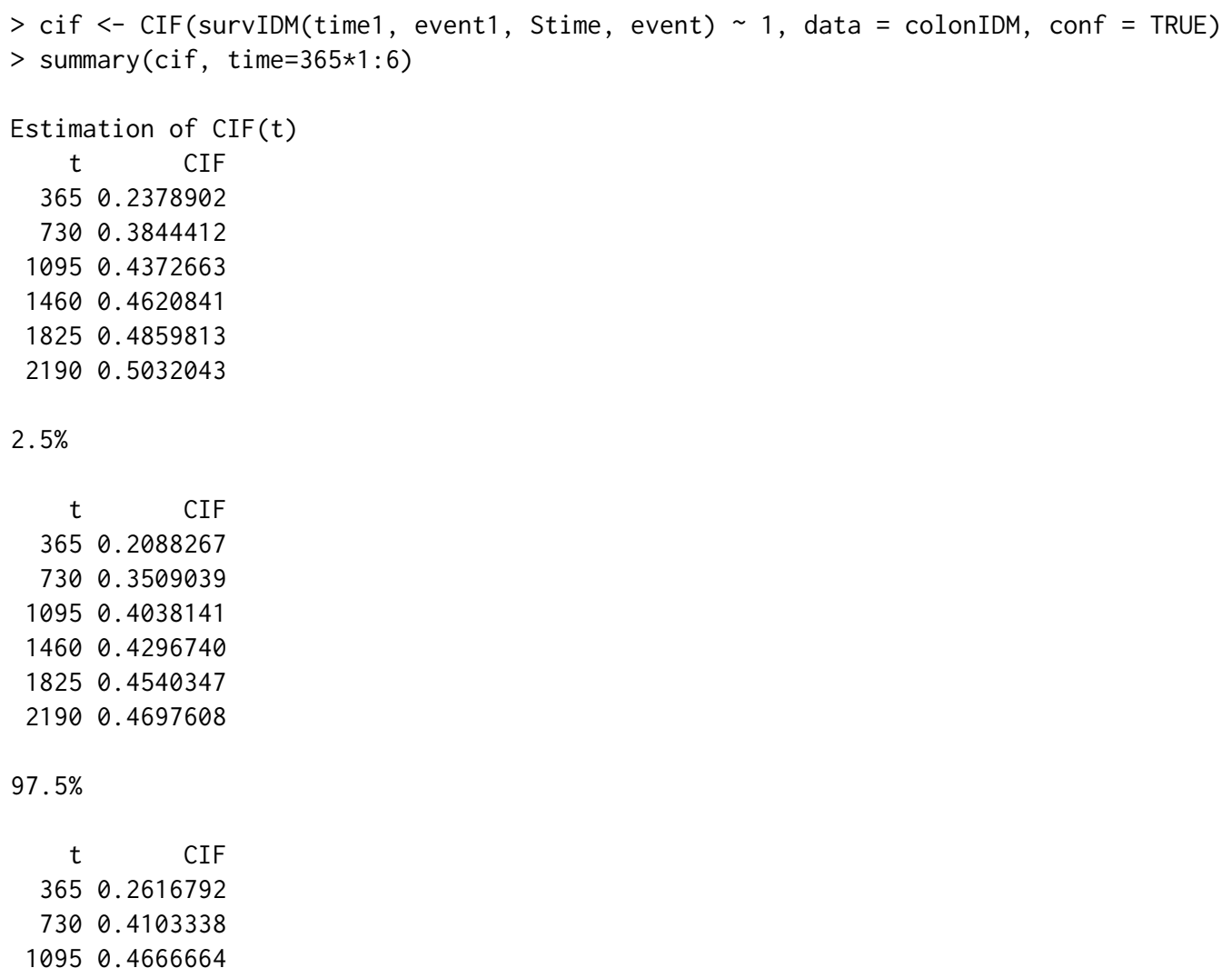




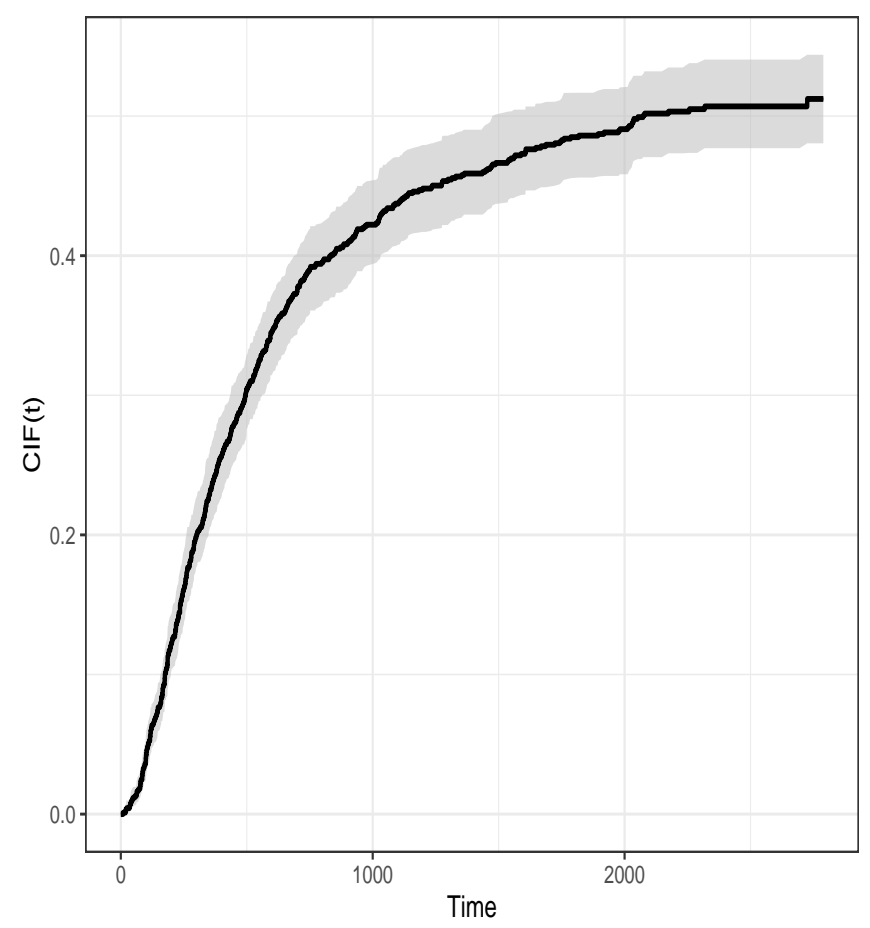

Figure 6: Cumulative incidence function in the recurrence state with $95 \%$ bootstrap confidence bands, using the colon cancer data.

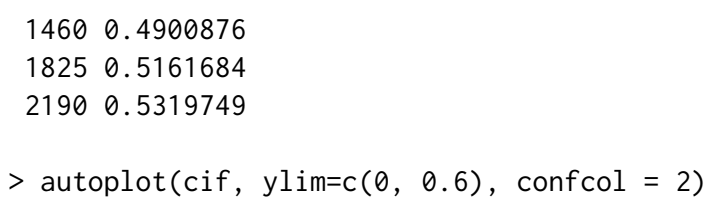

Figure 6 depicts the estimates of cumulative incidence function for the recurrent state together with a $95 \%$ pointwise confidence bands based on simple bootstrap that resamples each datum with probability $1 / n$. From this plot, it can be seen that individuals have a probability of recurrence higher than $50 \%$. This cumulative probability is about $43 \%$ at three years after surgery.

Figure 7 depicts the estimates of the (conditional) cumulative incidence function for patients with 1 and 9 lymph nodes with detectable cancer. Curves depicted in this figure, which are purely nonparametric, indicate that patients with 9 lymph nodes with detectable cancer have a considerably higher probability of recurrence. The corresponding input commands are shown below:

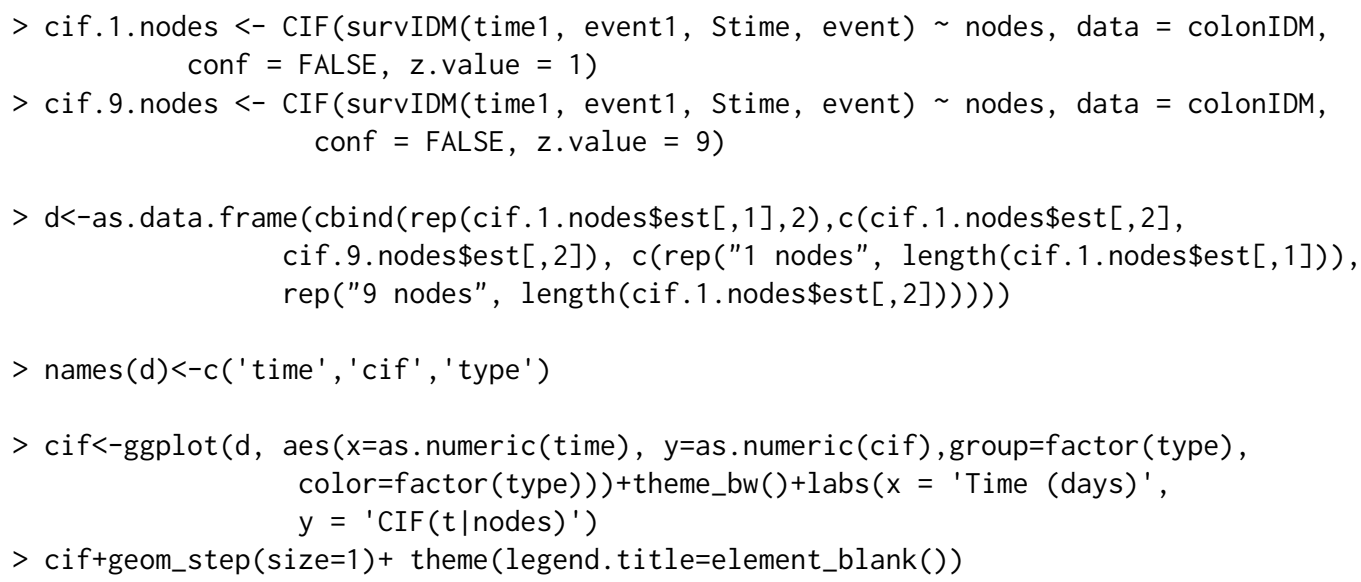




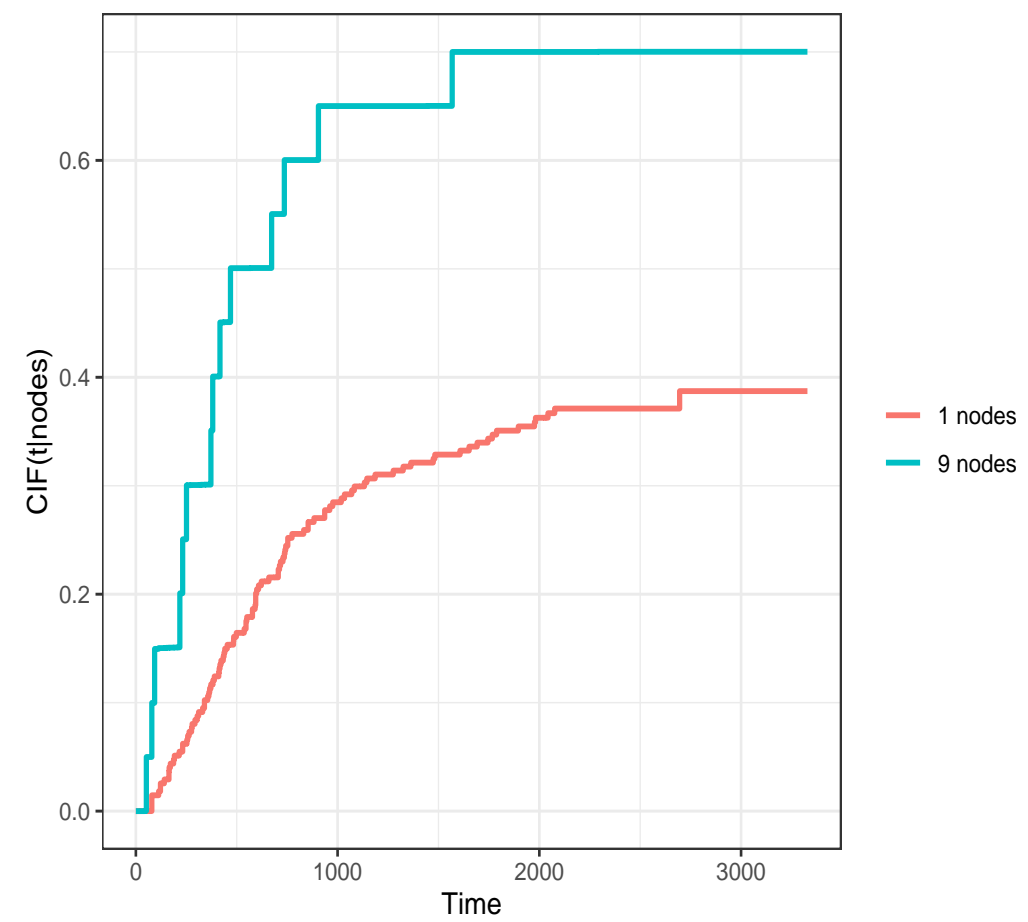

Figure 7: Conditional cumulative incidence function for the colon cancer data for nodes $=1$ and nodes $=9$, using the colon cancer data.

\section{Sojourn distribution}

Another interesting quantity is the sojourn time in each state. Estimates for the distribution function of the sojourn time in the recurrence state can be obtained using the estimator by Satten and Datta (2002) through function sojourn.

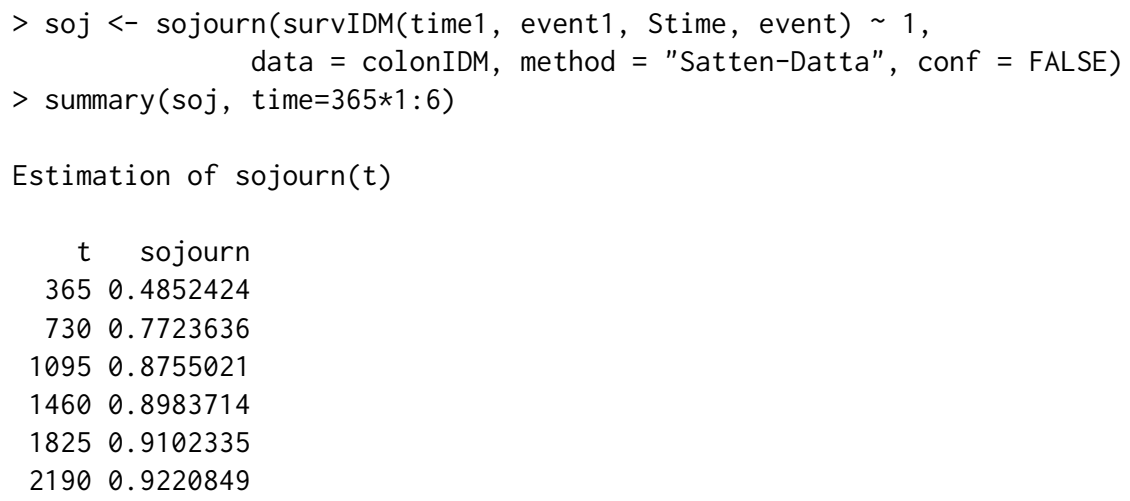

The estimates for the distribution function of the sojourn time in the recurrence state, corresponding to the time between entry in recurrence and death, reveal that the distribution function increases to a value near $49 \%$ and $78 \%$ for a time of one and two years, respectively, revealing a high risk of death shortly after relapse.

The methods for implementing some of the proposed methods can be computationally demanding. In particular, the use of bootstrap resampling techniques is time-consuming process because it is necessary to estimate the model a great number of times. In such cases, we recommend the use of parallelization (cluster = TRUE). This should considerably increase performance on multi-core/ multi-threading machines. 


\section{Conclusions}

There has been several recent contributions for the inference in the context of multi-state models. Many of these contributions were made for the illness-death model. One important and perhaps undervalued aspect of multi-state models is the possibility to apply them to obtain predictions of the clinical prognosis. This is usually achieved using estimates of the transition probabilities and survival estimates. However, there are several other quantities that could also be used in the analysis of these data, such as the state occupation probabilities, the sojourn time distributions, and the cumulative incidence functions. To provide the biomedical researchers with an easy-to-use tool for obtaining predictive estimates for all these quantities, we develop an $\mathrm{R}$ package called survidm. This package can be used to implement several nonparametric and semiparametric estimators for the transition probabilities. In addition, estimators have also implemented that account for the influence of covariates. Bootstrap confidence bands are provided for all methods. The software can also be used to perform multi-state regression (using type-specific Cox models).

One limitation of the survidm $\mathrm{R}$ package is that it can only be used in the progressive illness-death model. However, this turns out to be an advantage for those users that only wish to analyze data from a progressive illness-death model. For such cases, the survidm package is ideal since it is user-friendly (as illustrated in the real data analysis as well as in the help files of the main functions tprob, sojourn, and CIF) with a strong resemblance to the well-known and widely used survival package.

\section{Acknowledgments}

This research was financed by Portuguese Funds through FCT - "Fundação para a Ciência e a Tecnologia", within the research grant PD/BD/142887/2018. Luís Meira-Machado acknowledges financial support from the Spanish Ministry of Economy and Competitiveness MINECO through project MTM2017-82379-R funded by (AEI/FEDER, UE) and acronym "AFTERAM".

\section{Bibliography}

O. Aalen and S. Johansen. An empirical transition matrix for non homogeneous markov and chains based on censored observations. Scandinavian Journal of Statistics, 5:141-150, 1978. [p72]

O. O. Aalen, O. Borgan, and H. Fekjaer. Covariate adjustment of event histories estimated from markov chains: The additive approach. Biometrics, 57(4):993-1001, 2001. ISSN 0006-341X. [p72]

A. Allignol and A. Latouche. Cran task view: Survival analysis. Version 2018-05-04, URL http://CRAN.Rproject.org/view =Survival, 2018. [p71]

A. Allignol, M. Schumacher, and J. Beyersmann. Empirical transition matrix of multi-state models: The etm package. Journal of Statistical Software, 38:4:1-15, 2011. [p71]

P. K. Andersen, Ø. Borgan, R. D. Gill, and N. Keiding. Statistical Models Based on Counting Processes. Springer-Verlag, New York, 1993. [p70]

A. A. Araújo, J. Roca-Pardiñas, and L. Meira-Machado. Tpmsm: Estimation of the transition probabilities in 3-state models. Journal of Statistical Software, 62:1-29, 2014. [p71]

V. Balboa and J. de Uña-Álvarez. Estimation of transition probabilities for the illness-death model: Package tp.idm. Journal of Statistical Software, 83:10:1-19, 2018. [p71]

Ø. Borgan. Aalen-johansen estimator. Encyclopedia of Biostatistics, 1:5-10, 1988. [p72]

N. Breslow. Discussion of paper by dr cox. Journal of Royal Statistical Society, Series B, pages 216-217, 1972. [p73, 82]

R. Cao, I. Lopez-de Ullibarri, P. Janssen, and N. Veraverbeke. Presmoothed kaplan-meier and nelsonaalen estimators. Journal of Nonparametric Statistics, 17:31-56, 2005. [p72, 81]

D. Cox. Regression models and life tables. Journal of the Royal Statistical Society Series B, 34:187-220, 1972. $[\mathrm{p} 73,82]$

S. Datta and G. Satten. Validity of the aalen-johansen estimators of stage occupation probabilities and nelson aalen integrated transition hazards for non-markov models. Statistics \& Probability Letters, 55: 403-411, 2001. [p72, 73] 
J. de Uña-Álvarez and L. Meira-Machado. Nonparametric estimation of transition probabilities in the non-markov illness-death model: A comparative study. Biometrics, 71(2):364-375, 2015. ISSN 0006-341X. [p71, 72]

L. de Wreede, M. Fiocco, and H. Putter. mstate: An r package for the analysis of competing risks and multi-state models. Journal of Statistical Software, 38:7:1-30, 2011. [p71]

G. Dikta. On semiparametric random censorship models. Journal of Statistical Planning and Inference, 66:253-279, 1998. [p72, 81]

P. Eilers and B. Marx. Flexible smoothing with b-splines and penalties. Statistical Science, 11:89-121, 1996. [p77]

R. B. Geskus. Cause-specific cumulative incidence estimation and the fine and gray model under both left truncation and right censoring. Biometrics, 67(1):39-49, 2011. ISSN 0006-341X. [p73, 83]

P. Hougaard. Analysis of Multivariate Survival Data. Statistics for Biology and Health. Springer-Verlag, New York, 2000. [p70]

C. Jackson. Multi-state models for panel data: The msm package for r. Journal of Statistical Software, 38:8:1-28, 2011. [p72]

J. D. Kalbfleisch and R. L. Prentice. The statistical analysis of failure time data. John Wiley \& Sons, 1980. [p73]

E. Kaplan and P. Meier. Nonparametric estimation from incomplete observations. Journal of the American Statistical Association, 53:457-481, 1958. [p72]

L. Meira-Machado. Smoothed landmark estimators of the transition probabilities. SORT-Statistics and Operations Research Transactions, 40(2):375-398, Jul-Dec 2016. ISSN 1696-2281. [p72]

L. Meira-Machado and J. Roca-Pardiñas. p3state.msm: Analyzing survival data from an illness-death model. Journal of Statistical Software, 38:3, 2011. [p71]

L. Meira-Machado and M. Sestelo. Estimation in the progressive illness-death model: A nonexhaustive review. Biometrical Journal, 61:245-263, 2019. doi: 10.1002/bimj.201500038. [p70, 71, 73]

L. Meira-Machado, J. de Uña-Álvarez, and C. Cadarso-Suárez. Nonparametric estimation of transition probabilities in a non-markov illness-death model. Lifetime Data Analysis, 12:325-344, 2006. [p72]

L. Meira-Machado, J. de Uña-Álvarez, C. Cadarso-Suárez, and P. Andersen. Multi-state models for the analysis of time to event data. Statistical Methods in Medical Research, 18:195-222, 2009. [p70]

L. Meira-Machado, J. de Uña-Álvarez, and S. Datta. Nonparametric estimation of conditional transition probabilities in a non-markov illness-death model. Computational Statistics, 30:377-397, 2015. [p73]

C. G. Moertel, T. R. Fleming, J. S. Macdonald, D. G. Haller, J. A. Laurie, P. J. Goodman, J. S. Ungerleider, W. A. Emerson, D. C. Tormey, J. H. Glick, M. H. Veeder, and J. A. Mailliard. Levamisole and fluorouracil for adjuvant therapy of resected colon carcinoma. New England Journal of Medicine, 322 (6):352-358, 1990. [p74]

A. Moreira, J. de Uña-Álvarez, and L. Meira-Machado. Presmoothing the aalen-johansen estimator in the illness-death model. Electronical Journal of Statistics, 7:1491-1516, 2013. ISSN 1935-7524. [p72]

H. Putter and C. Spitoni. Non-parametric estimation of transition probabilities in non-markov multistate models: The landmark aalen-johansen estimator. Statistical Methods in Medical Research, 27: 2081-2092, 2018. [p73]

H. Putter, M. Fiocco, and R. B. Geskus. Tutorial in biostatistics: Competing risks and multi-state models. Statistics in Medicine, 26(11):2389-2430, mayo 2007. ISSN 0277-6715. [p70]

R Core Team. R: A Language and Environment for Statistical Computing. R Foundation for Statistical Computing, Vienna, Austria, 2019. URL https://www.r-project.org/. [p73]

M. Rodríguez-Álvarez, L. Meira-Machado, and E. Abu-Assi. Nonparametric estimation of timedependent roc curves conditional on a continuous covariate. Statistics in medicine, 35:7:1090-1102, 2016. [p73]

G. A. Satten and S. Datta. Marginal estimation for multi-stage models: waiting time distributions and competing risks analyses. Statistics in Medicine, 21(1):3-19, 2002. ISSN 0277-6715. [p73, 85] 
T. M. Therneau. A Package for Survival Analysis in R, 2021. URL https://CRAN.R-project.org/ package=survival. R package version 3.2-11. [p77]

H. C. Van Houwelingen. Dynamic prediction by landmarking in event history analysis. Scandinavian Journal of Statistics, 34(1):70-85, marzo 2007. ISSN 0303-6898. [p72]

M. Wand and M. Jones. Kernel Smoothing. Chapman \& Hal, London, 1997. [p81]

L. Wei. The accelerated failure time model: a useful alternative to the cox regression model in survival analysis. Statist. Med., 11:1871-1879, 1992. [p72] 
Gustavo Soutinho

Institute of Public Health of the University of Porto (ISPUP)

University of Porto

Rua das Taipas no. 135, 4050-600, Porto, Portugal

ORCID: 0000-0002-0559-1327

gdsoutinho@gmail.com

Marta Sestelo

Department of Statistics and OR,

SiDOR research group E CITMaga,

University of Vigo

Campus Lagoas-Marcosende, 36310 - Vigo, Spain

ORCID: 0000-0003-4284-6509

sestelo@uvigo.es

Luís Meira-Machado

Department of Mathematics \& Centre of Mathematics University of Minho

Campus de Azurém, 4800-058, Guimarães, Portugal

ORCID: 0000-0002-8577-7665

Imachado@math. uminho.pt 